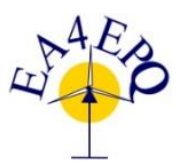

International Conference on Renewable Energies and Power Quality (ICREPQ'17) Malaga (Spain), $4^{\text {th }}$ to $6^{\text {th }}$ April, 2017

Renewable Energy and Power Qualing. Sournal (RE\&PQJ) ISSN 2172-038 X, No.15 April 2017

\title{
Design and Prototype of a Micro Hydrokinetic Vertical Turbine
}

\author{
A.M. Ramirez Tovar ${ }^{1}$, Y.U. Lopez ${ }^{2}$ and S.Laín ${ }^{2}$ \\ ${ }^{1}$ Renewable Energy for All-Foundation. $28^{\text {th }}$ Street \#100 Piamonte. Cali, Colombia \\ Phone: +57 3008622279, e-mail rtovar.am@gmail.com \\ ${ }^{2}$ Autonoma de Occidente University, Department of Energetic and Mechanic, \\ Km 2 via Cali-Jamundí, +572318 80 00, e-mail ylopez@uao.edu.co, slain@uao.edu.co
}

\begin{abstract}
In Latin America only few countries are 100\% connected to national electric networks. In Colombia, $52 \%$ of the national territory is known as NonInterconnected Zones with a population of 2 million people. To cover these zones basic electricity needs, the use of natural, local and renewable resources such as the hydraulic is proposed, due to the high energetic potential. For this purpose, there are several types of hydraulic turbines, most of them used for large scale power generation (UPME, 2010). This paper aims to design of hydraulic turbomachines for micro-generation to satisfy the electricity demand on isolated villages is deeply research. To accomplish this, a three straight blades Vertical Axis Micro Turbine H-Darrieus type is designed and modelled using CAD computational tools. Hydraulic characterization is run on a CFD ANSYS module and fluid-structure interaction is analyzed. Prototyping is made using 3D-printing process in a digital fabrication lab.
\end{abstract}

\section{KEYWORDS}

Micro-generation, vertical axis turbine, H-Darrieus.

\section{INTRODUCTION}

Globally, energy demand is growing and close to $40 \%$, with the higher levels in Asia (60\%) (IEA, 2014). Besides, rural electrification interest is growing and looking for technological solutions that use renewable energies in countries such as Indonesia, China, Nigeria and Brazil, among others (Chauhan y Saini, 2015), (Atmaja et al, 2014), (Shyu, 2012), (Olatomiwa et al, 2015), (Slough et al, 2015), (Shyu, 2012). Between renewables, solar, wind and hydro have increased its installed 2012 capacity up to $40 \mathrm{GW}$. $16,4 \%$ of this new power comes from hydropower technologies (REN21, 2014). Some of these technologies are classified in Axial (Horizontal) and Vertical (cross flow), Venturi and Vortex (Khan et al, 2009). In this research paper, the analysis of a design, model and manufacture of a micro H-Darrieus water turbine prototype is done. Moreover, its simulation using advanced computational tools considering a correlation between structure and fluid has been carried out 


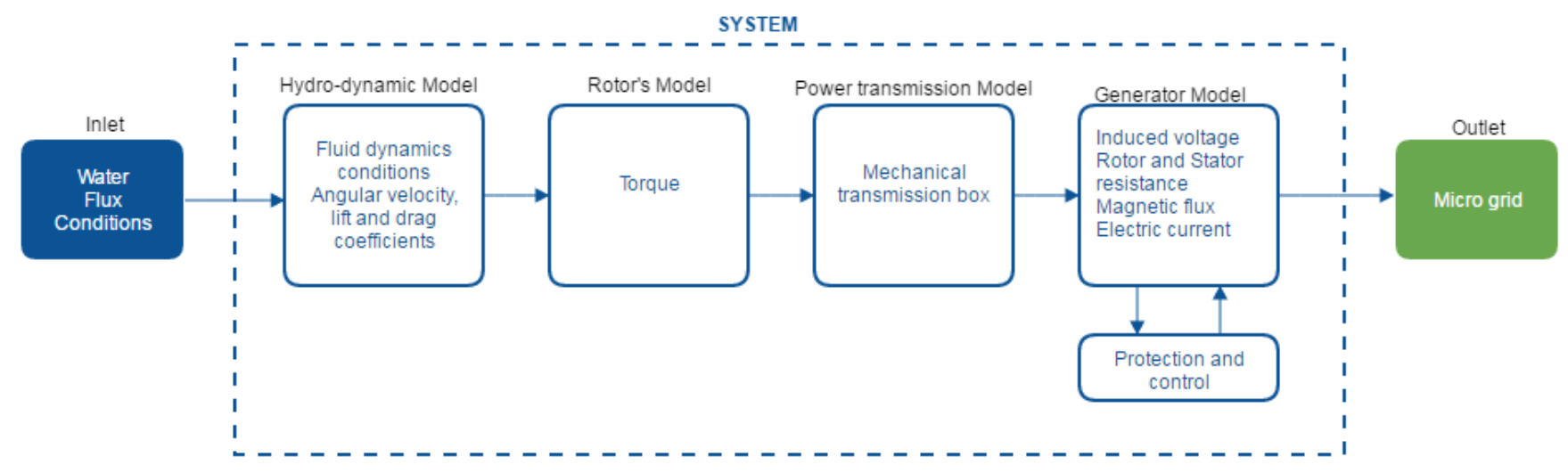

Figure 2. Model of the vertical axis hydrokinetic micro turbine system.

\section{MODEL OF A VERTICAL MICROTURBINE}

\section{Energy conversion}

Turbine gearbox and electric generator model are showed in figure 1 :

$$
E c=\frac{1}{2} m v^{2}
$$

Where:

$\mathrm{m}=$ water mass $(\mathrm{Kg})$.

$v=$ water velocity $(\mathrm{m} / \mathrm{s})$.

Mass flux of this water quantity:

$$
\dot{m}=\rho V
$$

For which:

$\rho$ : water density, $=1000 \mathrm{Kg} / \mathrm{m}^{3}$.

$V$ : cylinder's volume $\left(\mathrm{m}^{3}\right)$.

But, the volume of cylinder formed but the vertical axis turbine is:

$$
V=A l
$$

Where:

$A=$ sweeping area or surface $\left(\mathrm{m}^{2}\right)$

$l=$ cylinder length $(\mathrm{m})$

$A$ is the swept area or cross-section of the fluid, also known as the reference surface, and is estimated as shown in equation 4 , where $L$ is the blade length.

$$
A=2 R L
$$

To find a more detailed expression of the water kinetic energy, fluid mass is replaced by its equivalent in mass flow, thus obtaining:

$$
P_{\text {ext }}=\frac{1}{2} \rho A v^{3}
$$

Density, area and fluid velocity are related here.

There is a relationship called power coefficient, which determines the actual power available on the shaft, in such way:

$$
C_{p}=\frac{\text { Available power on the shaft }}{\text { Available power on the fluid }}
$$

$$
C_{P}=\lambda C_{m}
$$

Wherein:

$C_{m}=$ Torque or momentum coefficient (to the turbine) $\lambda=$ wing tip velocity:

$$
\lambda=\frac{r \omega}{V}
$$

For which:

$\omega=$ angular velocity $(\mathrm{rad} / \mathrm{s})$

$\mathrm{r}=\operatorname{radio}(\mathrm{mm})$

$v=$ instant linear water velocity $(\mathrm{m} / \mathrm{s})$

The power coefficient is an amount that expresses what fraction of the wind energy or water flow, is felt by the hydrokinetic turbine.

$$
E c=\frac{1}{2} \rho A V^{3} t \quad \text { (Joule) }
$$

The torque coefficient $C_{m}$ of the turbine is determined as: 


$$
C_{m}=\frac{\text { Torque }}{0,5 \rho v^{2} r A}
$$

By this way, theoretical power output on shaft is represented on the next equation.

$$
P=\frac{1}{2} \rho A v^{3} C_{p}
$$

\section{Power transmission system}

The power train system, allows transferring the mechanical water energy to the electric power generation system, as shown in figure 2 .

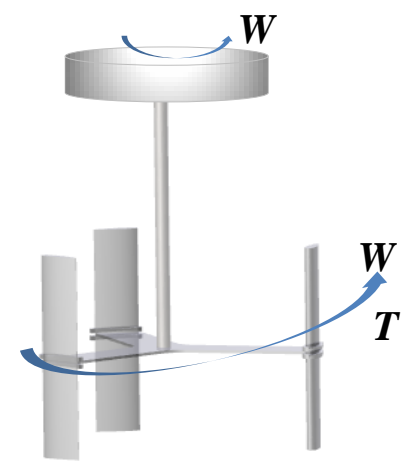

Figure 2. Vertical axis basic hydrokinetic micro turbine diagram.

Thus, the hydrodynamic force is transferred to the rotor, delivering a torsional torque to the electrical generator that has the same rotation direction. This system requires about $1800 \mathrm{rpm}$ to produce electricity at a nominal frequency to the connection point.

Only the torque of the turbine cannot generate the mechanical energy required by the electric generator. Therefore, sometimes it becomes necessary a transmission box, which raises the number of revolutions maintaining its torque.

In this research, an H-Darrieus vertical axis micro turbine, directly coupled to a permanent magnet generator (PMSG) has been modeled. This PMSG increases system reliability, reduces losses and decreases the system weight in the turbine-generator set (Samanvorakij and Kumkratug, 2013) (Haque et al, 2008) and (Hemeida et al, 2011).

Some manufacturers use direct connection generators, avoiding transmission boxes for small power systems. Others use gearboxes for low rpm reducers. There is a research confirming that the known Output Velocity Model is highly efficient [95\% -98\%], (Burton et al, 2001).

Once the micro-turbine is rotating, it produces a rotational speed $w_{T}$ and a torque $\tau_{\mathrm{T}}$, being inputs data to the gearbox. Then, this transmission box produces the generator input data : $w_{G}$ and $\tau_{G}$, as shown in equations $13-16$ :

$$
P m_{e} \cong P m_{s}
$$

$$
\omega_{T} \tau_{T}=\omega_{G} \tau_{G} \quad \text { To high efficiencies }
$$

To produce a higher speed at the output of the gearbox,

$$
\omega_{T} \ll \omega_{G}
$$

From equation 14, the output torque of the multiplier box is:

$$
\tau_{G}=N \tau_{T}
$$

Thus, the relation between generator and turbine speed

$$
N=\frac{\omega_{T}}{\omega_{G}}
$$

\section{Permanent Magnets Synchronous Generator.}

This kind of electrical rotating machine, could produce direct or altern current. Direct currents are recommended in short distances to maintain transmission and entire system efficiency (Layman, 1998) (Guney and Kaygusuz, 2010). The equivalent circuit modeling a permanent magnet synchronous generator, referred to the rotor, is shown in Figure 3. Moreover, the motion equation can be given by (Albernaz et al, 2015):

Where:

$$
\tau_{m}=\tau_{T}-\tau_{D}-\tau_{C}
$$

$\tau_{T}:$ Turbine torque

$\tau_{D}$ : Dissipation torque due to mechanical losses (friction)

$\tau_{C}$ : Equivalent load torque

The turbine torque and the equivalent load torque are given by equation (19) and (20), this is one indicates the generator torque (Albernaz et al, 2015).

$$
\tau_{T}=\frac{P}{\omega_{M}} \quad[N m]
$$




$$
\tau_{G}=\frac{1}{\eta} N * T_{T}[N m]
$$

Where:

$\mathrm{P}$ is the turbine output power on the shaft.

$\tau_{G}$ : Generator torque

$\eta$ : Transmission efficiency
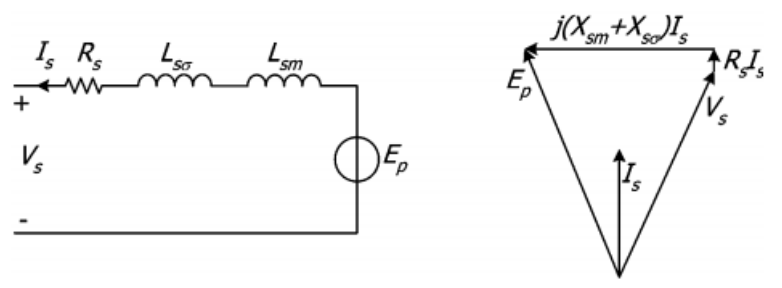

Figure 3. Equivalent circuit and phasor diagram of the permanent magnet electric generator. Source: (Polinder et al, 2006).

Where:

$I_{S}:$ Current Stator (A)

$R_{S}:$ Stator resistance $(\mathrm{m} \Omega)$

$L_{s \sigma}$ : Stator Dispersion Inductance $(\mathrm{mH})$

$L_{s}:$ Stator inductance $(\mathrm{mH})$

$V_{s}$ : Output Generator voltage or Source voltage

$E_{p}:$ Induced Voltage

Ep represents the stator voltage induced by the rotor permanent magnets. Thus, the generator performance in steady state is modelled by equation (21).

$$
V_{s}+I_{s} R_{s}+I_{s} j\left(X_{s \sigma} X_{s m}\right)=E_{p}
$$

\section{SIMULATION}

The design proposal is a vertical axis turbine, for low and medium flow rivers consisting of three symmetrical profiles NACA 0025, with a single central support as presented in figure 4 . Some other parameters can be appreciated on table 1 .

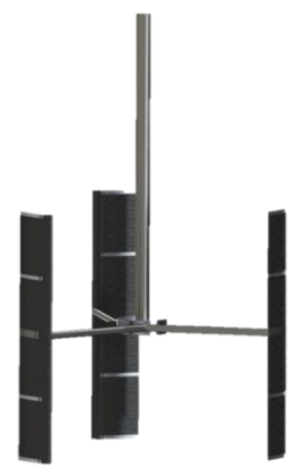

Figure 4. Design proposal
Table 1. Model features

\begin{tabular}{|l|l|}
\hline \multicolumn{1}{|c|}{ Electrical Variables } & Unit \\
\hline Source & \\
\hline Velocity $(\mathrm{m} / \mathrm{s})$ & $1,2-3$ \\
\hline Turbine & \\
\hline L: blade length $(\mathrm{mm})$ & 700 \\
\hline D. Diameter $(\mathrm{mm})$ & 900 \\
\hline C: Cord length $(\mathrm{mm})$ & 132.75 \\
\hline$\omega:$ constant angular velocity $(\mathrm{rad} / \mathrm{s})$ & 6.28 \\
\hline Generator & \\
\hline$L_{s}:$ Stator inducance $(\mathrm{mH})$ & 0.88 \\
\hline$L_{s \sigma}:$ Stator Dispersion $\mathrm{Inductance}(\mathrm{mH})$ & 1.44 \\
\hline$R_{s}:$ Stator resistance $(\mathrm{m} \Omega)$ & 22 \\
\hline
\end{tabular}

The geometric model is validated using numerical methods that allow a fluid-structure interaction through ANSYS simulation tool. The first simulation to be performed is CFX, where all the fluid analysis is developed.

To do this, two domains are created, the steady domain, which simulates the river movement of and the subdomain of smaller volumetric extension that represents the rotating part of the system (see figure 5). The dimensions of the computational domain are long (y) 20L, width (x) 13L and depth (z) 3.5L (Lain, Quintero, Trujillo, López, 2012).

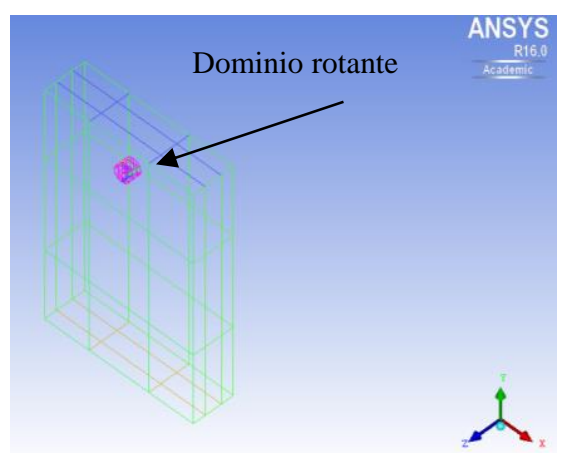

Figure 5. Combined Domains for CFX Simulation

The boundary conditions established for the simulation are shown in Table 2, while Figure 6 shows the regions where these conditions are applied. In the structural evaluation phase, it is simulated at a speed of $3 \mathrm{~ms}-1$ 
representing the upper limit to establish the critical operating conditions; However, the speed of operation is in $1.2 \mathrm{~ms}-1$

Table 2. Simulation border conditions in CFX

\begin{tabular}{|l|l|}
\hline Element & Condition \\
\hline Inltet & Uniform flux $3 \mathrm{~ms}^{-1}$ \\
\hline Outlet & Pressure: $0 \mathrm{~Pa}$ \\
\hline Wall & Free slip Wall \\
\hline Turbine & No slip wall \\
\hline
\end{tabular}

Once the boundary conditions are determined, all the variables to be exported in the fluid-structure interaction (pressure on the solid reference of the mesh) are monitored. After obtaining simulation results in CFX, pressure field are exported as initial value to the ANSYS Structural module.

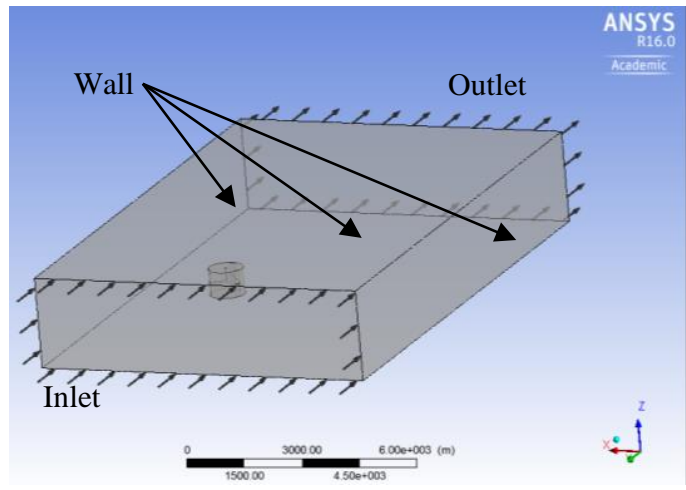

Figure 6. CFX simulation - boundary conditions

Since the simulation purpose is to validate the proposed geometry, a second mesh is made, which represents only the mechanical elements as shown in figure 7. With this mesh, the second part of the simulation by finite elements is developed. Since, it analyses the mechanical effects like deformations, stresses and mechanical vibrations (among others) that the fluid medium generates on the turbine by the interaction.

The only turbine contour condition is the fixed support at top of the shaft. The above condition is determined based on the assumption that the axis is in cantilever and the other surfaces have no other support, it is in free interaction with the previously simulated fluid.

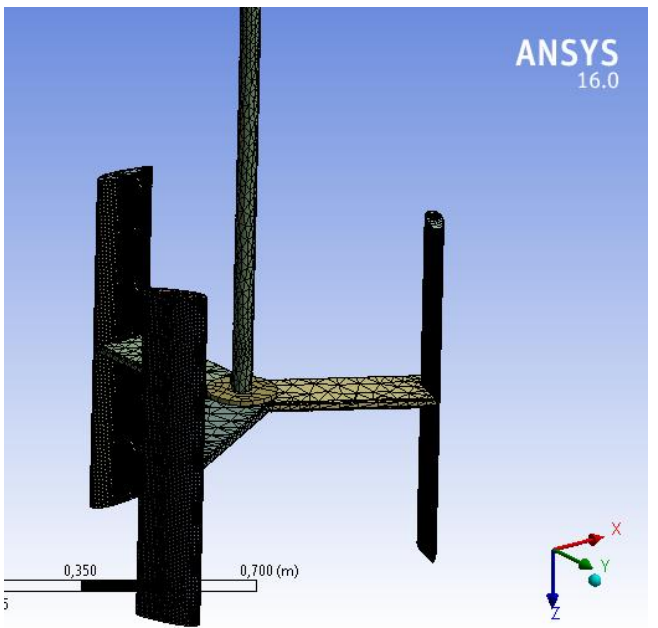

Figure 7. Structural mesh

Materials selection plays an important role to determine stresses reference parameter determination. Three parameters are taken for choice, the first is Young's density-modulus ratio, since it is required to be resistant but low weight materials (for buoyancy purposes); The second parameter is the price, meanwhile it is necessary to be accessible to the target people in rural zones, and the last selection parameter is the primary $\mathrm{CO} 2$ production.

Using the Granta CES 2016 software, comparative graphics are made for different materials families. Finnally, aluminium T84 is chosen due to its internal profiles and reinforcements and because it has a good resistance performance to fatigue in corrosive agents (M. Guérin, J. Alexis, E. Andrieu, C. Blanc, \& G. Odemer, 2015); Stainless steel for the shaft and a carbide - silicon compound to coat the profile and give the NACA shape.

\section{RESULTS}

Due to the CAD and ANSYS used for simulation, some issues were encountered in the mesh's performance because of topological compatibility; therefore, it was necessary to re-configure turbine geometry in CAD and to make the mesh on the exterior surfaces of this. 


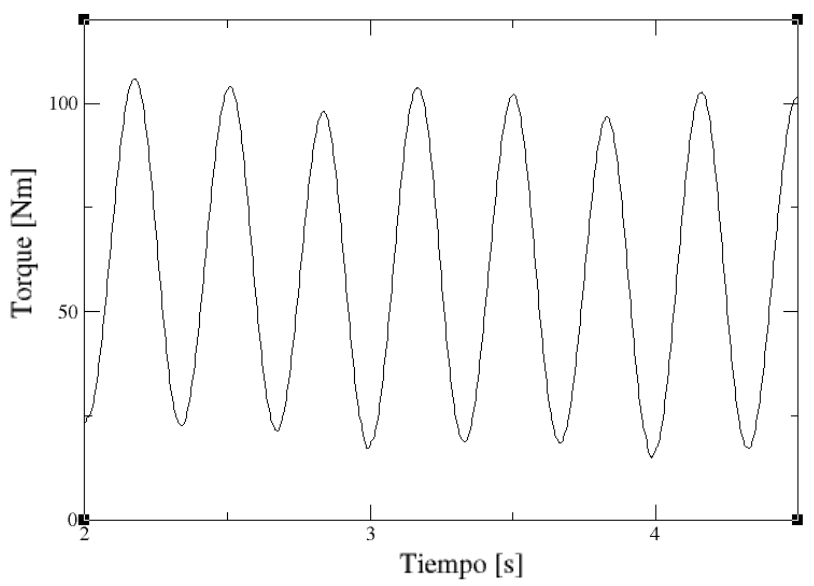

Figure 9. Torque

Figure 9 shows the torque behavior along the turbine revolution, reaching a maximum torque around $100-\mathrm{Nm}$ and a minimum of $20-\mathrm{Nm}$, for an average of $60-\mathrm{Nm}$ of generation, which is equivalent to a $380 \mathrm{~W}$ output power.

\subsection{Pressure and velocity fields}

Pressure and velocity are compared from three different and sequential positions, on a plane $\mathrm{x}$ and at a height of $1.49 \mathrm{~m}$ on the z-axis. To clarify perspectives shown, an orange circle has been painted on number one blade in figure 10 , specifying that in position 1 , this is $90^{\circ}$ to zero location. In addition, in position 2 to $180^{\circ}$ of the same. These provisions are chosen because they allow exposing three states that would be considered structurally critical.

In the position 0, central axe (support) has an arm completely parallel to the fluid, but a perpendicular blade. In the opposite case, one arm of this support is perpendicular to the fluid and to the parallel profile. In 2 , performance and angle are similar to that in position 0 , but inverted to the fluid.

\section{Pressure}

It is appreciated that the value of the static pressure tends to be homogeneous and constant. Position 2 demonstrates that pressure behavior tends to be the same as other locations, however, blade three, which is completely perpendicular to the fluid, supports a pressure increase almost over all its extension, representing a possible critical condition from a structural point of view.

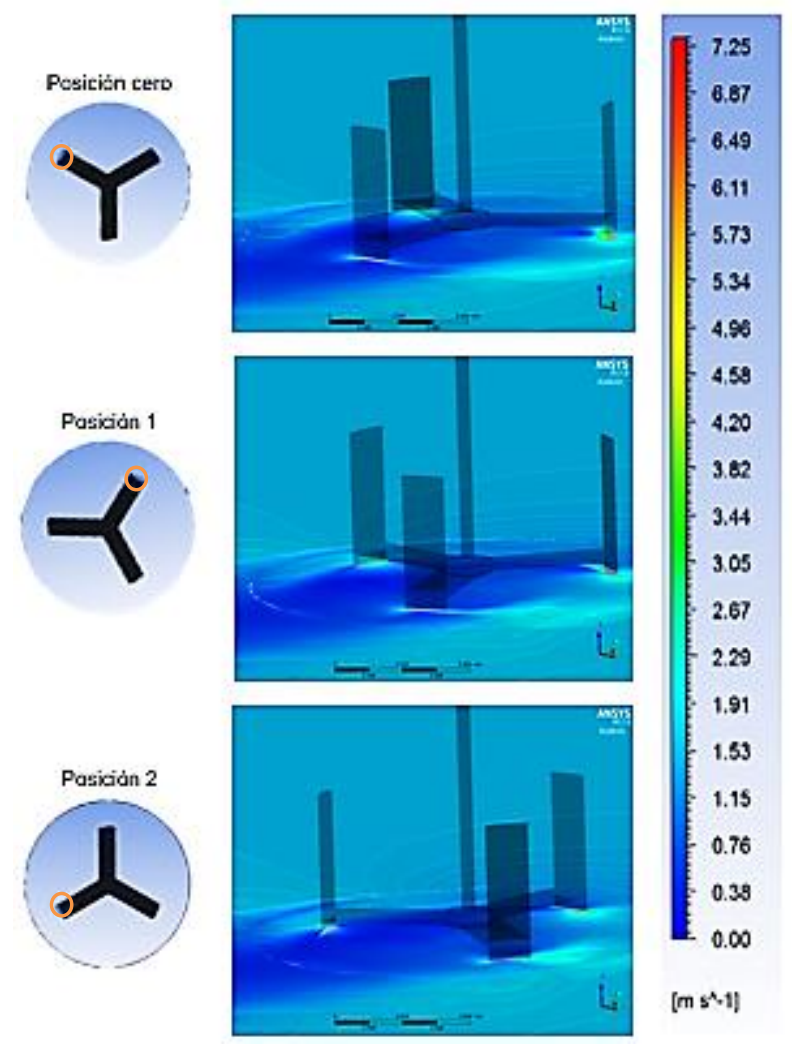

Figure 10. Critic positions into velocity field

\section{Velocity}

Behind the turbine, a wake is formed generating a decrease of velocity and is related to the thrust coefficient, since this determines the extracted flow moment, in other words: kinetic energy has been turn from the medium and transformed into mechanical energy.

As the fluid velocity increases downstream, the gradient between the wake and free flow forms an additional turbulence, which facilitates momentum transfer to the surrounding fluid.

It can also be seen that upstream, there is a velocity decrease in fluid on turbine surroundings, due to the structure.

\subsection{Deformations}

Due to fluid dynamic interaction, figure 11 shows structure deformations in a transient regime. There is a maximum of 2.3-mm deformation presented in the lower part of the blade, that represents $0.33 \%$ of the total NACA profile length. 


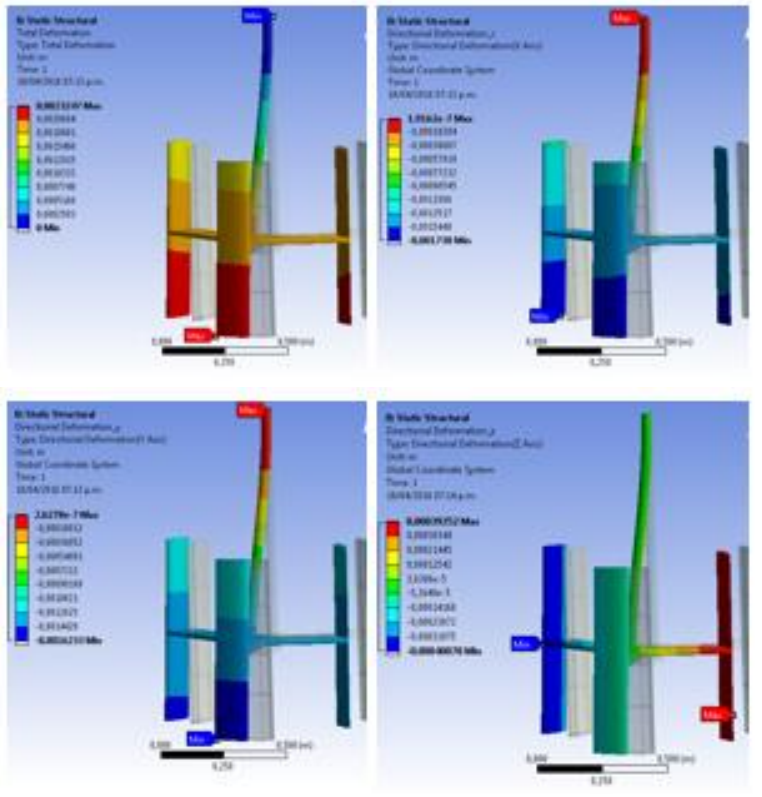

Figure 11. Deformation

This result ensures that structure do not have buckling problems. Since this analysis is performed by taking a linear fluid velocity of $200 \%$ over the rated speed, it is guaranteed that when it reaches the critical value $\left(3 \mathrm{~ms}^{-}\right.$ ${ }^{1}$ ) it will not fail due to static problems or fatigue deformation. In the same way, deformations are analyzed on each axis, verifying there is not risk condition.

On the coordinate axis $x$ and $y$, in the lower part of the blades a maximum deformation value is observed; however, deformations are not worrying to be 1,9 and 1,6-mm respectively (Figure 11). On the other hand, the maximum deformation on $\mathrm{z}$ is less than $1 \mathrm{~mm}$ which indicates that does not represent a risk either.

\section{CONSTRUCTION}

With the support of Laboratory of Digital Fabrication FABLAB Cali, and using mechanical and aerodynamical parameters, a prototype is done. The first one at 1:10 scale, is made using a $3 \mathrm{D}$ printing technique in a Machine Maker Bot with ABS polymer, as shown in figure 12 .

A full-scale blade is made in balance with the rib construction technique, as shown in figures 13 and 14 .

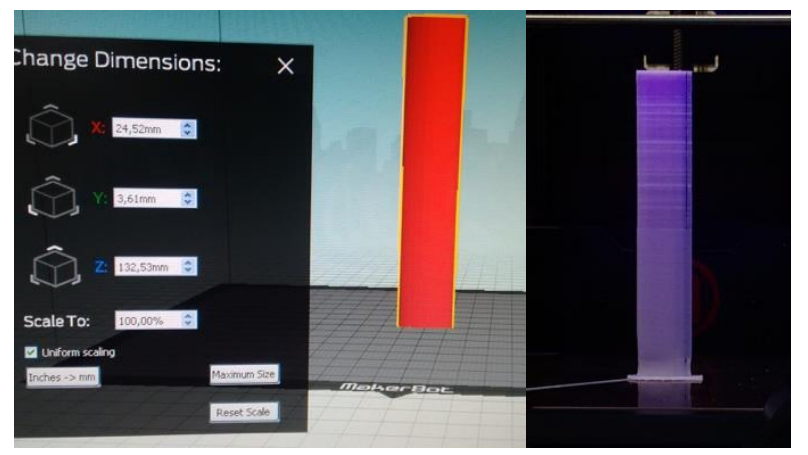

Figure 12. Prototype 3D printed

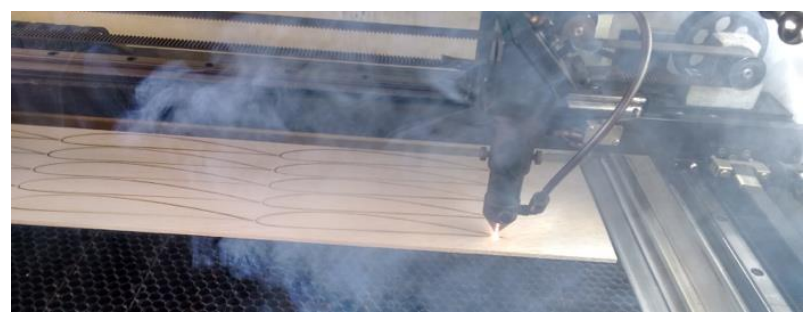

Figure 13. Laser cutting of NACA 0025 profiles

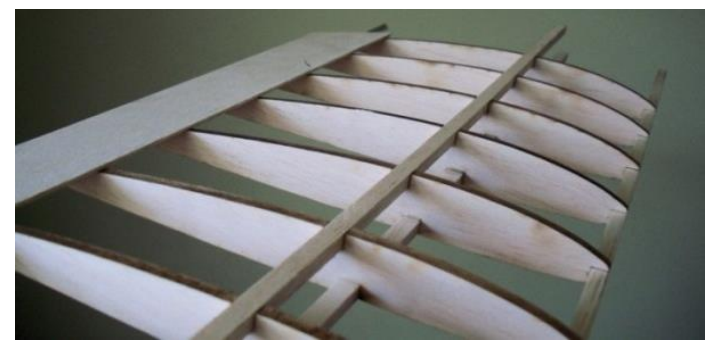

Figure 14. Blade construction in balso wood using ribs technique.

\section{CONCLUSIONS}

- Recent research publications have worked on vertical axis water turbines, but just few focused on designing. However, there isn't evidence of research on designing, modeling and prototyping. Hence, this research article presents the design criteria, modeling part and prototyping of a H-Darrieus vertical axis hydrokinetic micro turbine system for rural applications producing $380 \mathrm{~W}$ of peak power (simulated). Output power is approximately $400-\mathrm{W}$, represented by an angular velocity of 60 -rpm and a generated torque of 54-Nm. According to his low output power, this machine is classified as a peakcentral generation.

- On this research process, several hydrodynamic models were analyzed to predict an optimal turbine performance of a hydrokinetic H-Darrieus microturbine design. Thus, this process defines turbine 
materials, yielding that Aluminum T84 is ideal, due to its good mechanical properties against abrasive environments (Ocean implementation).

\section{REFERENCES}

Unidad de Planeación Minero Energética, UPME CORPOEMA. Formulación Plan de Desarrollo FNCE [En línea]. Disponible en http://www.upme.gov.co/Sigic/Informes/Informe_A vance 01.pdf . 2010.

IEA. World Energy Outlook 2014. International Energy Agency IEA. O D/IEA. http://www.iea.org/ 2014.

A. Chauhan, R.P. Saini, Renewable energy based offgrid rural electrification in Uttarakhand state of India: Technology options, modelling method, barriers and recommendations, Renewable and Sustainable Energy Reviews, Volume 51, November 2015, Pages 662-681.

S. Atmaja P. Rosyidi, Tjasa Bole-Rentel, Surya Budi Lesmana, Jazaul Ikhsan, Lessons Learnt from the Energy Needs Assessment Carried out for the Biogas Program for Rural Development in Yogyakarta, Indonesia. Procedia Environmental Sciences, Volume 20, 2014, Pages 20-29

C. Shyu. (2012). Rural electrification program with renewable energy sources: An analysis of China's Township Electrification Program. Energy Policy, 51, 842-853.

L. Olatomiwa, Saad Mekhilef, A.S.N. Huda, Olayinka S. Ohunakin, onomic evaluation of hybrid energy systems for rural electrification in six geo-political zones of Nigeria, Renewable Energy, Volume 83, November 2015, Pages 435-446.

T. Slough, Johannes Urpelainen, Joonseok Yang, Light for all?. Evaluating Brazil's rural electrification progress, 2000-2010, Energy Policy, Volume 86, November 2015, Pages 315-327.

M. J. Khan, G. Bhuyan, M.T. Iqbal, J.E. Quaicoe, Hydrokinetic energy conversion systems and assessment of horizontal and vertical axis turbines for river and tidal applications: A technology status review, Applied Energy, Volume 86, Issue 10. October 2009.

T. Burton., D. Sharpe., N. Jenkins., \& E. Bossanyi. (2001). Wind energy handbook. John Wiley \& Sons.

H. Polinder, Van der Pijl, F. F., Vilder, D., \& Tavner, P. J. (2006). Comparison of dir t-drive and geared generator concepts for wind turbines. Energy conversion, IEEE transactions on, 21(3), 725-733.

M. S. Güney, K. Kaygusuz, Hydrokinetic energy conversion systems: A technology status review, Renewable and Sustainable Energy Reviews, Volume 14, Issue 9, D ember 2010, Pages 29963004.
J. Albernaz, Jerson Rogério Pinheiro Vaz, Alexandre Luiz Amarante Mesquita, André Luiz Amarante Mesquita, Claudio José Cavalcante Blanco, An Approach for the Dynamic Behavior of Hydrokinetic Turbines, Energy Procedia, Volume 75, August 2015, Pages 271-276

S. Laín, B. Quintero, D. Trujillo, Y. Ulianov, Simulation of Vertical Axis Water Turbines, IEEE, 2012.

S. Samanvorakij, P. Kumkratug. "Modeling and Simulation PMSG based on Wind Energy Conversion System in MATLAB/SIMULINK. Proc. of the Second Intl. Conf. on Advances in Electronics and Electrical Engineering-AEEE. 2013.

M. E. Haque, M. Negnevitsky, K. M. Muttaqi, A novel control strategy for a Variable Speed Wind Turbine with a Permanent Magnet Synchronous Generator. IEEE Transaction on Industry Applications, Vol 46, No 1, pp. 1-8, 2008.

A. M. Hemeida, W. A. Farag, O. A. Mahgoub. Modeling and Control of Direct Driven PMSG for Ultra Large Wind Turbines. World Academy of Science Engineering and Technology. Vol. 59, pp. 621-627, 2011.

REN21. 2014. Global Status Report. Paris: REN21 Secretariat. 